\title{
Abducens Nerve Palsy Caused by Basilar Impression Associated with Atlanto-occipital Assimilation
}

\author{
-Case Report-
}

\author{
Yuichi Hirose, Masachika SagoH, Keita MaYanagi, and Hideki MuraKami
}

Department of Neurosurgery, Ashikaga Red Cross Hospital, Ashikaga, Tochigi

\begin{abstract}
A 47-year-old male presented with abducens nerve palsy due to basilar impression associated with atlanto-occipital assimilation manifesting as slowly progressive bilateral trigeminal neuralgia and diplopia in the right lateral gaze. X-ray and computed tomography of the skull confirmed the diagnosis of basilar impression and atlanto-occipital assimilation, and magnetic resonance imaging disclosed tightness of the posterior cranial fossa. Surgical suboccipital decompression resulted in gradual resolution of the patient's complaints, and no additional symptoms were recognized. Impairment of the sixth cranial nerve is a rare symptom compared to those of the fifth or the eighth cranial nerve in a patient with a craniocervical malformation. However, the present case shows the possibility of cranial nerve dysfunction due to tightness of the posterior cranial fossa, and suggests that surgical treatment for basilar impression with atlanto-occipital assimilation should be considered in patients with uncommon and unusual symptoms.
\end{abstract}

Key words: abducens nerve palsy, basilar impression, atlanto-occipital assimilation, posterior decompression

\section{Introduction}

Atlanto-occipital assimilation is a malformation of the craniocervical junction characterized by a bony union between the occiput and atlas and is sometimes associated with other cranial or vertebral anomalies. " Atlanto-occipital assimilation itself is considered to be asymptomatic other than limitation of the movement of the neck, but coexistent anomalies may cause various neurological disorders. We present a case of basilar impression associated with atlanto-occipital assimilation. The patient complained of slowly progressive unilateral abducens nerve palsy. In the reported cases, this symptom is uncommon in anomalies of the craniocervical junction, ${ }^{3,14)}$ however, in the present case, the symptoms were relieved after surgical decompression of the posterior cranial fossa, and tightness of the fossa due to atlanto-occipital assimilation and basilar impression was considered to have caused impairment of the sixth cranial nerve. This report may well offer assistance regarding the decision on the correct operative indication for patients with similar anoma-

Received October 9, 1997;

Accepted March 9, 1998 lies suffering from symptoms which are uncommon in previous studies.

\section{Case Report}

A 47-year-old male had suffered nuchal pain or dullness for 10 years, but had received no particular treatment other than oral administration of analgesics. He had developed pain in his forehead and bilateral cheeks one year previously, and noticed diplopia in the right lateral gaze 2 months prior to admission to our hospital.

On admission, neurological examination revealed bilateral trigeminal hyperalgesia in the territories of the first and the second branches of the nerve and was more pronounced on the right side. The facial pain was lancinating, and was both spontaneous and induced by tapping on the patient's forehead and cheeks, i.e., "trigger points" were present. Diplopia associated with right abducens nerve palsy was confirmed by the incomplete movement of the right eyeball in the full lateral gaze to the right. Nystagmus was not recognized. The other cranial nerves were not involved and the patient showed no cerebellar symptoms. Deformity or shortness of the neck was 


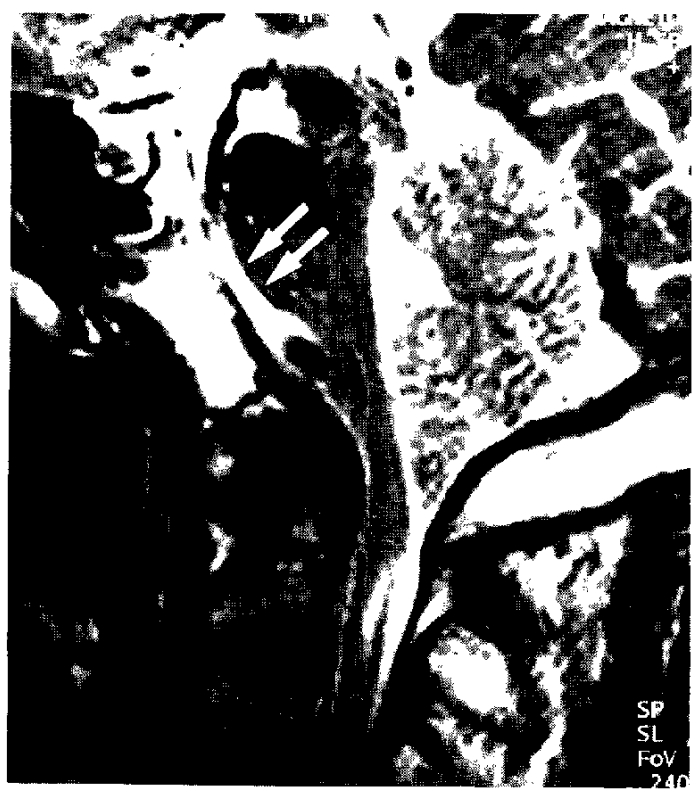

Fig. $1 \mathrm{~T}_{2}$-weighted magnetic resonance image showing ventral compression of the cervicomedullary junction by the odontoid process of the axis and syringomyelia at the C-1 to C-2 levels. The posterior arch of the atlas cannot be differentiated from the occiput, and the fusioned bone is thick and compresses the caudal portion of the cerebellum. The space between the clivus and brain stem is small (arrows).
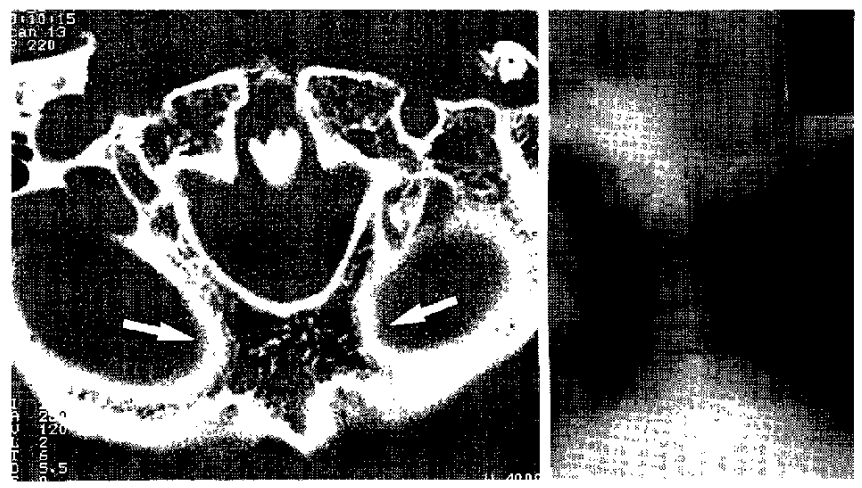

Fig. 2 Computed tomography scan (left) and x-ray tomogram (right) of the skull confirming that the posterior arch of atlas is fused with the occiput (arrows).

not found, but movement of the neck was slightly limited when hyperflexion or hyperextension was intended. No additional symptoms dependent on the posture were present. Standard blood and urine chemistry examinations found nothing unusual.

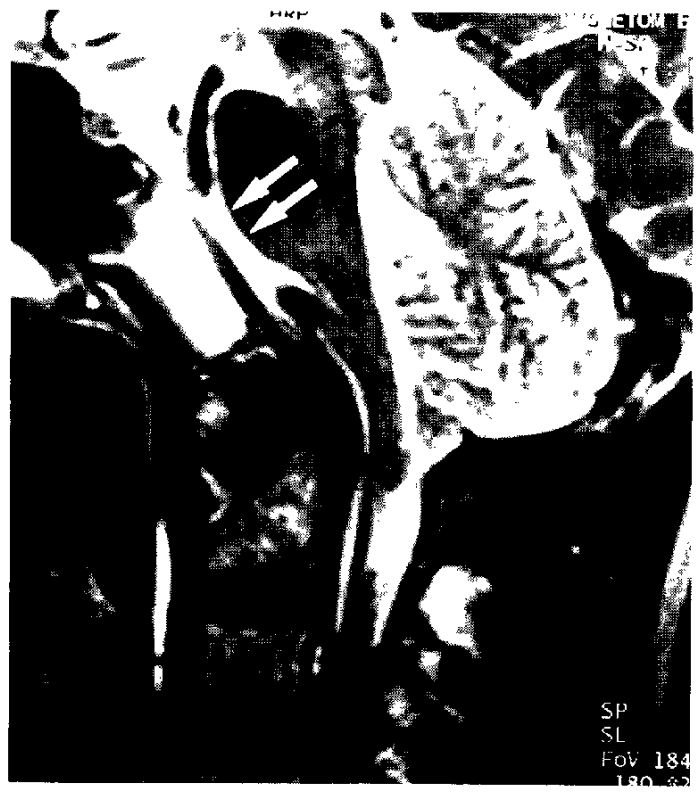

Fig. 3 Postoperative $T_{2}$-weighted magnetic resonance image showing reduced dorsocaudal compression of the cerebellum and enlarged space between the clivus and brain stem compared to the preoperative image (arrows).

Magnetic resonance (MR) imaging showed ventral compression of the cervicomedullary junction by the odontoid process of the axis and syringomyelia at the C-1 to C-2 levels (Fig. 1). In addition, the posterior arch of the atlas could not be differentiated from the occiput, and the thick fused bone had compressed the caudal portion of the cerebellum, which was tightening the posterior cranial fossa. No ventricular dilatation was seen. Computed tomography (CT') and x-ray tomography of the skull confirmed the diagnosis of atlanto-occipital assimilation associated with basilar impression (Fig. 2). Platybasia was not recognized and the other vertebrae were radiologically normal. X-ray tomography of the craniocervical junction with the patient's neck flexed and extended showed that the distance between the anterior arch and the odontoid process of the axis did not change regardless of the posture, thus ruling out atlanto-axial dislocation. Neither MR imaging nor CT disclosed any abnormal lesion in the brain stem which could have caused abducens nerve palsy. Cerebral angiography found no abnormality. CT myelography revealed that the lower medulla was compressed ventrally by the odontoid process of axis and the surrounding connective tissues, and that there was communication between the cisterns.

Although the cause of the abducens nerve palsy 
was not clarified, suboccipital craniectomy was performed to reduce compression in the structures in the posterior cranial fossa, because this surgical procedure could be of benefit in preventing progression of the syringomyelia. ${ }^{3.11}$ Operative findings showed the occiput fused with the atlas was very thick, but the dura mater and the structures in the subdural space showed no abnormal morphology.

After the operation, the patient did not develop any additional symptoms and movement of the neck was smooth. Diplopia in the right lateral gaze and facial hyperalgesia resolved gradually, and postoperative MR imaging showed that the dorso-caudal compression of the cerebellum was evidently reduced and the brain stem shifted dorsally, which resulted in enlargement of the space between the clivus and brain stem (Fig. 3). The patient has been followed up for 12 months, and all his preoperative complaints have resolved.

\section{Discussion}

The clinical and symptomatic characteristics of atlanto-occipital assimilation depend on any coexistent anomalies. The present case was associated with basilar impression, and the medulla was compressed by the odontoid process of the axis. This condition might be involved in various neurological and pathological mechanisms. Anterior compression of the medulla has caused neurogenic hypertension as well as impairments of lower cranial nerves, which illustrates how uncommon neurological symptoms can be caused by basilar impression. ${ }^{5)}$ However, in our patient, direct neuronal compression by the odontoid process was not thought to be the reason for the abducens nerve palsy because the compression was localized in the lower medulla, where neither nervous fibers nor nuclei associated with the sixth cranial nerve are present. The lower part of the cerebellum was compressed by the assimilated atlas, but this also could not be directly responsible for his complaints. His abducens nerve palsy resolved after suboccipital craniectomy, so it could have originated from some compressive force to the nervous tissue, but there was no lesion directly affecting the sixth cranial nerve. Vertebral artery dissection has been related to basilar impression, ${ }^{61}$ and we also suspected the possibility of some vascular abnormality causing the abducens nerve dysfunction, for example, arterial tortuosity which could compress the associated nerve. However, angiography showed no abnormal findings and MR imaging revealed no ischemic focus in the brain stem.

Preoperative MR imaging in the present case revealed that the lower part of the cerebellar tonsil was located in front of the posterior arch of the atlas, which was assimilated with the occiput bone, and that the posterior cranial fossa was constricted. These findings have something in common with Chiari I malformation, ${ }^{21}$ and our patient's symptoms developed in adulthood in the same way. Chiari I malformation exhibits various neurological disorders including impairments of cranial nerves. ${ }^{3,8,14)}$ The trigeminal nerve is the most commonly affected cranial nerve and is occasionally associated with dysfunction of the eighth to twelfth cranial nerves. Impairment of the lower cranial nerves may be due to traction,,$^{3)}$ although this hypothesis does not account for the abducens nerve palsy, and this dysfunction, at least without impairment of lower cranial nerves, is not included in the neurological symptoms reported in a large series of the patients with Chiari I malformation. Abducens nerve palsy occurred after the rupture of a superior cerebellar artery aneurysm in a patient with a meandering basilar artery. ${ }^{10)}$ Therefore, increased intracranial pressure, or a minor shift of the intracranial structures, can cause such nerve dysfunction in a patient with pre-existing factors. In the present case, the space between the midbrain and the clivus was smaller, and this might be the determinant for the abducens nerve palsy during the clinical course of atlanto-occipital assimilation associated with basilar impression. Various lesions can cause acquired abducens nerve palsy, for example, trauma, neoplasm, aneurysm, and vascular compression. ${ }^{15]}$ However, the most common etiology is "unknown," and the classification as "vascular" is purely speculative and based merely on the presence of existing vasculopathy or risk factors and the lack of any other neurological signs. ${ }^{15)}$ A case of posterior fossa tumor presented with trigeminal and abducens nerve disorders as false localizing signs, ${ }^{13)}$ possibly due to vascular compression on these nerves resulting from the distortion of the brain stem. In our case, no parenchymal lesion was seen in the posterior fossa, but postoperative MR imaging showed a minor dorsal shift of the brain stem accompanied by improvement of the patient's symptoms. Therefore, vascular compression might have been the cause of the trigeminal and abducens nerve disorders, although this could not be proven. The abducens nerve emerges from the ventral surface of the pons (pontomedullary sulcus) and runs through the osteofibrous Dorello's canal before reaching the cavernous sinus. ${ }^{7)}$ The nerve is located inside a venous confluence which occupies the space between the dural leaves of the petroclival area, and is only separated from the venous blood by the meningeal sheath which is sometimes very thin. ${ }^{16)}$ This may explain 
the cause of abducens nerve palsy in patients with increased venous pressure, ${ }^{4}$ such as carotid cavernous fistula. In fact, about half of the patients with carotid cavernous fistula also suffer from abducens nerve palsy. ${ }^{12)}$ However, there was no evidence of intracranial venous hypertension in our patient.

The mechanism causing abducens nerve palsy in our patient remained unclear, but surgical decompression of the posterior cranial fossa should be considered for the treatment of patients with atlanto-occipital assimilation and/or basilar impression associated with unusual or uncommon symptoms. ${ }^{9)}$

\section{References}

1) Bertrand G: Anomalies of the craniovertebral junction, in Youmans JR (ed): Neurological Surgery, ed 2. Philadelphia, WB Saunders, 1982, pp 1484-1486

2) Chiari H: Über Veränderungen des Kleinhirns infolge von Hydrocephalie des Grosshirns. Dtsch Med Wochenschr 17: 1172-1175, 1891 (Ger)

3) Cristante L, Westphal M, Hermann HD: Cranio-cervical decompression for Chiari I malformation. A retrospective evaluation of functional outcome with particular attention to the motor deficits. Acta Neurochir (Wien) 130: 94-100, 1994

4) Destrieux C, Velut S, Kakou MK, Lefrancq TL, Arbeille B, Santini JJ: A new concept in Dorello's canal microanatomy. The petro-clival venous confluence. I Neurosurg 87: 67-72, 1997

5) Dickinson LD, Papadopoulos SM, Hoff JT: Neurogenic hypertension related to basilar impression. Case report. J Neurosurg 79: 924-928, 1993

6) Dickinson LD, Tuite GF, Colon GP, Papadopoulos SM: Vertebral artery dissection related to basilar impression: Case report. Neurosurgery 36: 835-838, 1996

7) Dorello P: Considerazion sopra la causa della paralisi transitoria dell'abducente nelle flogosi dell'orecchio medio. Atti Clin Otorinolaringoiatrica Univ Roma 3: 207-217, 1905 (Ital)

8) Elster AD, Chen MYM: Chiari I malformations: Clinical and radiologic reappraisal. Radiology 183: 347353,1992

9) Erbengi A, Oge HK: Congenital malformations of the craniovertebral junction: Classification and surgical treatment. Acta Neurochir (Wien) 127: 180-185, 1994

10) Hirose $Y$, Nakamura T, Takamiya $Y$, Kinoshita N, Hirai H: Fusiform superior cerebellar artery aneurysm presenting with contralateral abducens nerve paresis. Case report. Neurol Med Chir (Tokyo) 30: 119-122, 1990

11) Kohno K, Sakaki S, Nakamura H, Sakoh H, Takeda S, Sadamoto K: Foramen magnum decompression for syringomyelia associated with basilar impression and Chiari I malformation. Report of three cases. Neurol Med Chir (Tokyo) 31: 715-719, 1991

12) Lewis AI, Tomsick TA, Tew JM Jr: Management of 100 consecutive direct carotid-cavernous fistulas. Result of treatment with detachable balloons. Neurosurgery 36: 239-245, 1995

13) Ro LS, Chen ST, Tang LM, Wei KC: Concurrent trigeminal, abducens, and facial nerve palsies presenting as false localizing signs. Case report. Neurosurgery 37: 322-325, 1995

14) Sclafani AP, DeDio RM, Hendrix RA: The Chiari-I malformation. Ear Nose Throat J 70: 208-212, 1991

15) Tiffin PAC, MacEwen CJ, Craig EA, Clayton G: Acquired palsy of the oculomotor, trochlear and abducens nerves. Eye 10: 377-384, 1996

16) Umansky F, Elidan J, Valarezo A: Dorello's canal. A micro-anatomical study. J Neurosurg 75: 294-298, 1991

Address reprint requests to: Y. Hirose, M.D., Department of Neurosurgery, Ashikaga Red Cross Hospital, 32100 Honjo, Ashikaga, Tochigi 326-0808, Japan. 